\title{
The Normal Zone Propagation in ATLAS B00 Model Coil
}

\author{
E. W. Boxman, A. V. Dudarev, and H. H. J. ten Kate
}

\begin{abstract}
The B00 model coil has been successfully tested in the ATLAS Magnet Test Facility at CERN. The coil consists of two double pancakes wound with Aluminum stabilized cables of the Barrel- and End-Cap Toroids conductors for the ATLAS detector. The magnet current is applied up to $24 \mathrm{kA}$ and quenches are induced by firing point heaters. The normal zone velocity is measured over a wide range of currents by using pickup coils, voltage taps and superconducting quench detectors. The signals coming from various sensors are presented and analyzed. The results extracted from the various detection methods are in good agreement. It is found that the characteristic velocities vary from 5 to $20 \mathrm{~m} / \mathrm{s}$ at 15 and $24 \mathrm{kA}$ respectively. In addition, the Minimum Quench Energies at different applied magnet currents are presented.
\end{abstract}

Index Terms-MQE, NPZ, stabilized, superconductivity.

\section{INTRODUCTION}

$\mathbf{T}$ HE CONSTRUCTION of the $1 \mathrm{~m}$ size B00-coil is completed and successfully tested in the Atlas test facility [1]. A minimum temperature of $4.8 \mathrm{~K}$ is reached on the coil by indirect cooling and the maximum magnet current of $24 \mathrm{kA}$ is applied. The later is the limit of the $6 \mathrm{~V}$ power supply. All subsystems including the two service turrets, the interface for the cryogenic system and the power system are checked. The coil consists of two different double pancakes. One uses a former version of the Barrel Toroid cable (BT) and the other uses the EndCap Toroid (ECT) cable. Each pancake contains 10 layers of conductor Details of the conductors and coil are in reference [2].

Here is suffices to recall the main characteristics. The BT conductor has overall dimensions of the Aluminum of $57 \times 12 \mathrm{~mm}^{2}$ and it contains a 32 strand Rutherford cable with dimensions of $25 \times 2.3 \mathrm{~mm}^{2}$, positioned in the center. The critical current is $65 \mathrm{kA}$ at $5 \mathrm{~T}$ while the conductor operates in a magnetic peak field of $2.6 \mathrm{~T}$ at $24 \mathrm{kA}$ in the inner most coil winding of B00. The ECT conductor is similar, $40 \times 12 \mathrm{~mm}^{2}$ with an operating current of $24 \mathrm{kA}$ in a $2.9 \mathrm{~T}$ peak field. During the several quench tests, the ECT coil and the BT coil were connected in series. Due to the large amount of Aluminum around the NbTi superconductor, calculations of the normal zone velocity are strongly influenced by the current diffusion effect. The latter depends on the purity of the Aluminum and therefore depending on the magnetic field value. Together with the number of parameters involved in the calculations, it makes precise calculations of the normal zone velocity difficult to attain.

Manuscript received September 24, 2001. This work was supported in part by the Netherlands Technical Foundation (STW).

E. W. Boxman is with the University of Twente in the Netherlands and CERN/Switzerland Geneva 23, CH-1211 (e-mail: Hubert.Boxman@ @ern.ch).

A. V. Dudarev and H. H. J. ten Kate are with CERN/Switzerland Geneva.

Publisher Item Identifier S 1051-8223(02)04235-5.

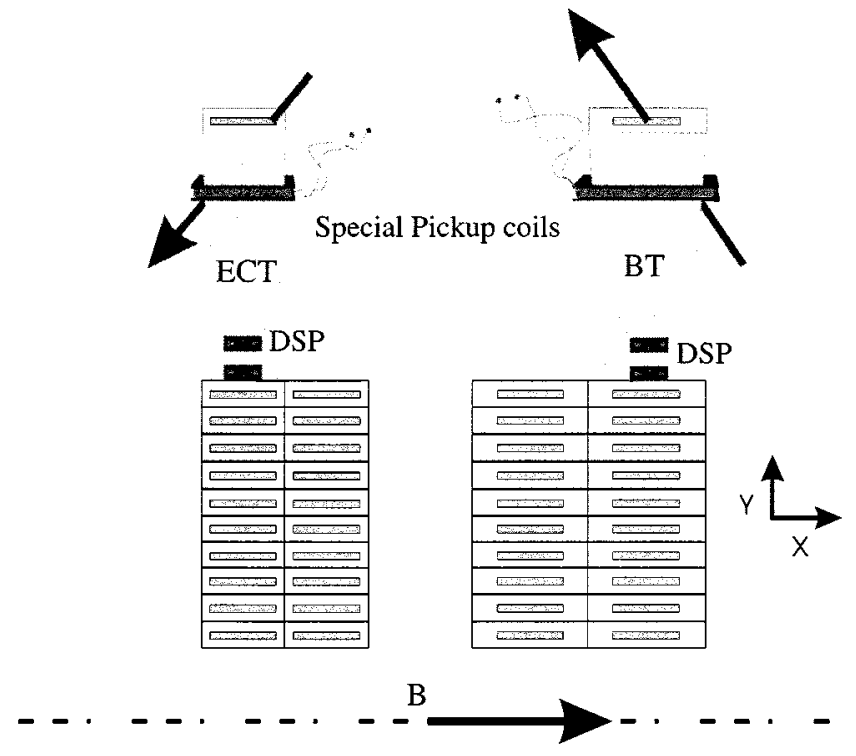

Fig. 1. The position of the special pickup coil and two out of the 20 Diffusion Solenoid Pickup coils in B00 model coil for the Atlas Magnet System. The current leads are connected close to the special pickup coil.

\section{Signals From Pickup CoILS DuRING A NORMAL TRANSITION EVENT}

When a normal transition event takes place in the Al-stabilized superconductors, the current is forced to go from the $\mathrm{NbTi}$ strands into the Aluminum cladding. During this current diffusion, a flux change can be detected with a solenoid pickup coil, situated just outside the conductor [4]. Another effect of flux change is due to the Rutherford shape of the cable. The shape can also be seen as a long solenoid coil. If there is a normal transition, the magnetic flux inside the Rutherford cable changes to zero. As this is the only flux component in the longitudinal direction of the cable, neglecting global field disturbances, the signal noise ratio is expected to be high in the case the magnet current is kept constant. In order to detect the mentioned flux change, two special pick-up coil are developed with typically 600 windings. They are placed around the conductor as depicted in Fig. 1.

\section{A. Copyright Form}

In Fig. 1, the conductor position of one half of the B00-coil is presented. The position of the special pickup coils, two Diffusion Solenoid Pickup coils (DSP) and the direction of the magnetic field (arrows) are schematically shown. 


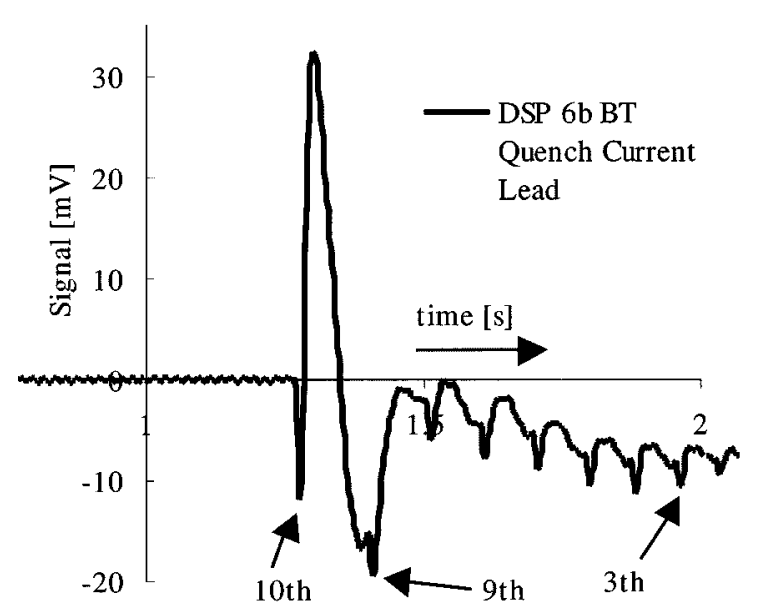

(a)

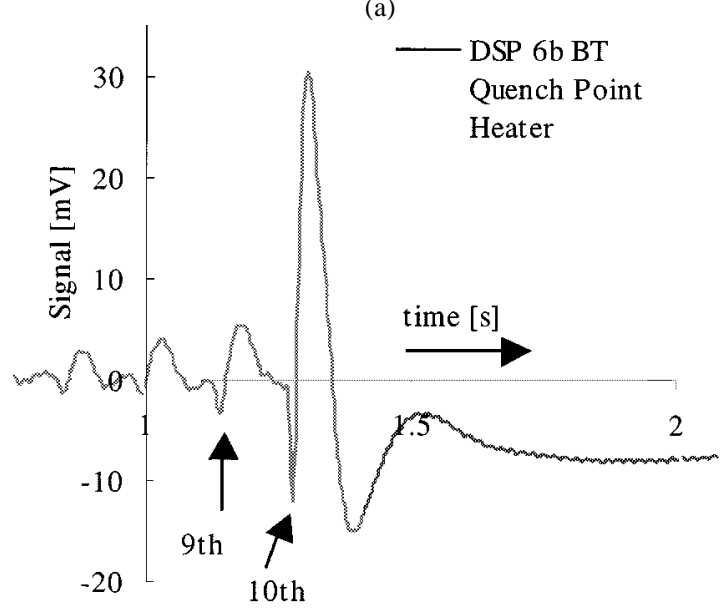

(b)

Fig. 2. The Diffusion Solenoid Pickup coil (DSP) signals during the normal transition. (a) The quench starts from the current lead. (b) The quench is initialized by the point heater.

\section{B. Diffusion Solenoid Pickup Coil}

Two signals from the DSP's during normal transition are presented in Fig. 2(a) and (b). The sensors are situated on top of the last layer of the BT coil on the most outer pancake as shown in Fig. 1.

The first signal is measured when the indirect cooling of the current lead is stopped with a magnet current of $20.5 \mathrm{kA}$. In this case, the normal zone starts from outside the coil, propagating toward the first turn. The second signal is measured when the normal zone starts at the quench heater on the most inner turn, propagating toward the current lead with a magnet current of $20 \mathrm{kA}$. The first signal is shifted in time in such a way that the largest peak starts at the same time in both cases.

Both signals have similar behavior. When the normal zone passes the pickup coil, a small negative signal ( 0 to $20 \mathrm{~ms}$ ) followed by a larger positive signal (20 to $100 \mathrm{~ms}$ ) can be seen. This is a result of a relatively fast flux change at the normal zone front. The reason behind this effect is not understood. After this flux change, the diffusion of the current into the Aluminum starts (typically $5 \mathrm{~s}$. in the current range of 5 to $24 \mathrm{kA}$ ). Two diffusion effects can be distinguished. First the current diffuses in the $y$-direction as described in Fig. 1. This is a faster process compared to the second process, the diffusion in the $x$-direction,

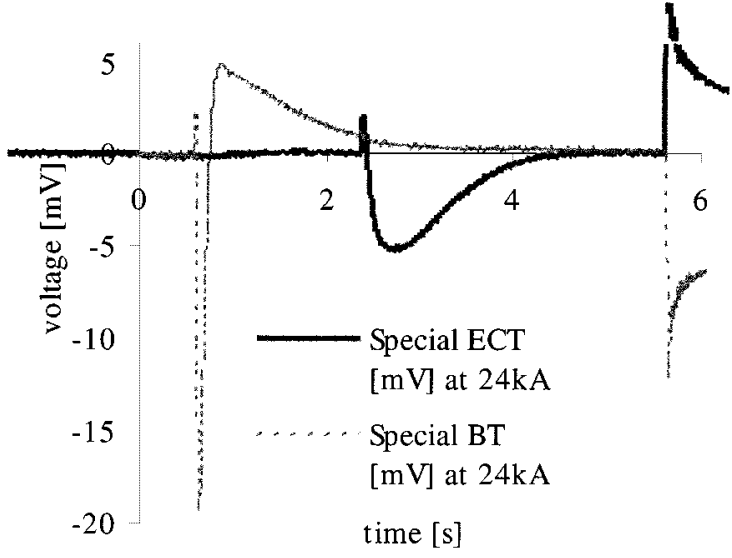

Fig. 3. The special pickup coil signals during the normal transition event with a constant magnet current of $24 \mathrm{kA}$.

simply due to the shorter diffusion length. The characteristic time constants of the second process are determined for magnet currents between 7 to $24 \mathrm{kA}$ of the BT and the ECT conductor from the DSP's situated on the last turns of the ECT and BT coil in B00. The results are the same order of magnitude as the expected characteristic time constant for diffusion, considered as a one-dimensional diffusion problem [3]. The DSP is also sensitive to the flux change as a result of the normal transition inside the other layers. On top of the main signal, the layers that are before/after quenching are indicated with arrows in the figures. It also shows the dominating longitudinal quench propagation. This is expected, as the characteristic time constant of the radial heat conduction through the insulation layer, is smaller than the time for the normal zone to reach the next layer. In this way, using only one sensor the normal zone propagation velocity can be determined in all of the layers with reasonable accuracy. It is found that pickup coils that are situated on the inner pancake do not show this effect as strongly as presented here. This suggests that the gaps in the cover plate of B00 [2] make it possible to transfer the flux change in one layer over a large distance $(>100 \mathrm{~mm}$ ), while for the inner pancake the flux is screened by the coil casing.

\section{Special Pickup Coils}

In Fig. 3, the signals are represented of the two special pickup coils during a quench. The normal zone was initialized by firing the point heater situated on the most inner turn of the BT coil at around $t=0 \mathrm{~s}$. The magnet current was kept constant at $24 \mathrm{kA}$ until 5.7 seconds. At this moment, the Magnet Safety System (MSS) discharges the magnet $(\tau \approx 0.75 \mathrm{~s})$. The MSS has a delay of 5 seconds between the detection and the discharge. The detection is done by super-conducting quench detectors (SQD's) situated a few centimeters from the special pickup coils. During the discharge, the induced voltage signals from the special pickup coils are in the opposite direction as expected from the magnetic field map, as schematically shown in Fig. 1.

At 0.7 seconds the front of the normal zone in the BT conductor at the position of the special pickup coil (Special BT) is detected. After $2.4 \mathrm{~s}$. the zone has also quenched the complete ECT coil and the front is at the position of the ECT special pickup coil (Special ECT). The first large induced voltage 


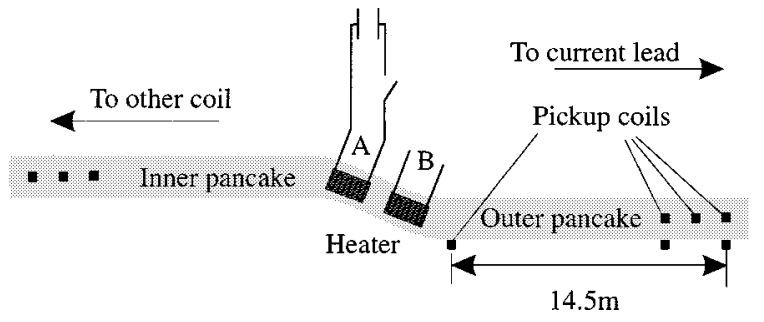

Fig. 4. Schematic view of the pick-up coil position and heaters inside the B00 model coil. The current lead is connected to the outer pancake.

is in the opposite direction as well as for the ECT as for the BT special pickup coil. The duration of this flux change is roughly $0.1 \mathrm{~s}$. This suggests that it can not come from the flux change of the twist inside the individual strands as normally this would be on the order of $10 \mathrm{~ms}$. [5]. However, with the additional Aluminum cladding the time that is needed for the decay of these kind of "rotating" currents could be larger.

After the first peak, the diffusion process of the Rutherford component of the current is decaying in the Aluminum. Taking the integral of the induced voltage, the characteristic time constant of the diffusion process can be calculated. At $24 \mathrm{kA}$, the characteristic time constant is $0.95 \mathrm{~s}$ and $0.48 \mathrm{~s}$ for the BT and the ECT conductor, respectively. Analyses of the signals at different applied magnet currents, show a very high $\mathrm{S} / \mathrm{N}$ ratio ( $>100$ at $10 \mathrm{kA}$ ). Also, there is no direct contact with the coil itself. Together, this passive coil/sensor makes it suitable for quench protection systems.

\section{NORMAL ZONE PROPAGATION}

The normal zones are initialized by discharging a set of capacitors $\left(V_{\max }=600 \mathrm{~V}, 730 \mu \mathrm{F}\right)$ over the flat point heaters $(10 \Omega)$ directly glued on the surface of the conductors on the most inner turn. Special care is taken to assure good thermal contact between the heater and the surface of the conductor. The time constant of the heater pulse is less than $4.5 \mathrm{~ms}$. This is below the suggested characteristic formation time of the minimum propagation zone (50-100 ms) and therefore the energy needed to quench the system no longer depends on the pulse duration [6]. At different applied magnet currents up to $24 \mathrm{kA}$, quenches are initialized and the velocity of the normal zone front is calculated from the time information between two pickup coil signals and the distance of the pickup coils. The first pickup coil is situated $50 \mathrm{~mm}$ away from the position of the heater, which is situated on the most inner turn of the magnet. The second sensor is situated on the most outer layer of the coil. Along the turns this gives a relative distance of $14.5 \mathrm{~m}$ between the two pickup coils, as schematically shown in Fig. 4.

Therefore, an average normal zone velocity is determined concerning all the layers. The energy necessary to create a NPZ is carefully increased, in such a way that the normal zone is created at the minimum quench energy (MQE). It is found that the amount of energy did not influence the average normal zone velocity. Also firing heater A or B or A and B parallel did not influence the normal zone velocities. The results of the normal velocities depending on the applied magnet current are presented in Fig. 5 for the BT conductor as well as for the ECT conductor. The characteristic velocities vary from 5 to $20 \mathrm{~m} / \mathrm{s}$ at

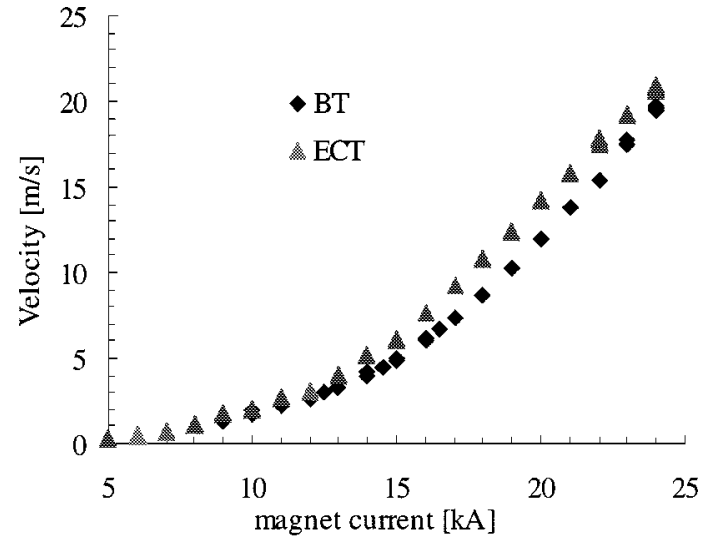

Fig. 5. The average longitudinal normal zone propagation velocity depending on the magnet current in the B00 coil. Rectangular and square symbols are the velocities inside the BT- and the ECT conductor, respectively.

15 and $24 \mathrm{kA}$ respectively. Despite the higher critical current in the ECT, which results in a higher sharing temperature, the velocity of the normal zone front is larger in the ECT conductor above $13 \mathrm{kA}$. This can be understood, considering that the larger amount of stabilizer is improving the cooling power of the BT conductor.

In addition, when the current diffuses into the Aluminum cladding the ohmic dissipation is less in the BT conductor as the Aluminum cross-section is larger. Together this results in a higher propagation velocity in the ECT conductor. At low magnet currents $(<15 \mathrm{kA})$ the amount of energy that is needed to create the quench increases rapidly. The time necessary for the normal zone front to reach the next layer in the pancake becomes comparable to the radial heat diffusion time constant. This implies that the initial temperature is already increased in the layers before the front of the normal zone passes. However, it never exceeded the current sharing temperature before the layer was quenched. Therefore, a transverse normal zone propagation has not been detected in the B00 coil.

\section{Minimum QUENCH ENERGY}

In Fig. 6(a) and (b) the minimum quench energies vs. the applied magnet currents are presented of the BT conductor and the ECT conductor, respectively. The quenches are induced with the same point heaters situated on the most inner turn of the two coils as described in the normal zone propagation. The time for the heat to reach the superconductor is determined by the isolation layer between the heater and the conductor. In this time a part of the heat could flow in another direction and not contribute to the MQE. Estimation of this amount of heat show that an error less than $0.1 \mathrm{~J}$ is made, which is far below the MQE values in the figures. Analyzing the results, three regions can be distinguished.

- No Signal: The coil voltage as well as the pickup coil signals showed no signal that could indicate that there is a diffusion of the current into the Aluminum cladding. The normal conducting part of the conductor is smaller than the distance between the pickup coil and the heater and the coil tension is lower than the limit of the data acquisition system. 


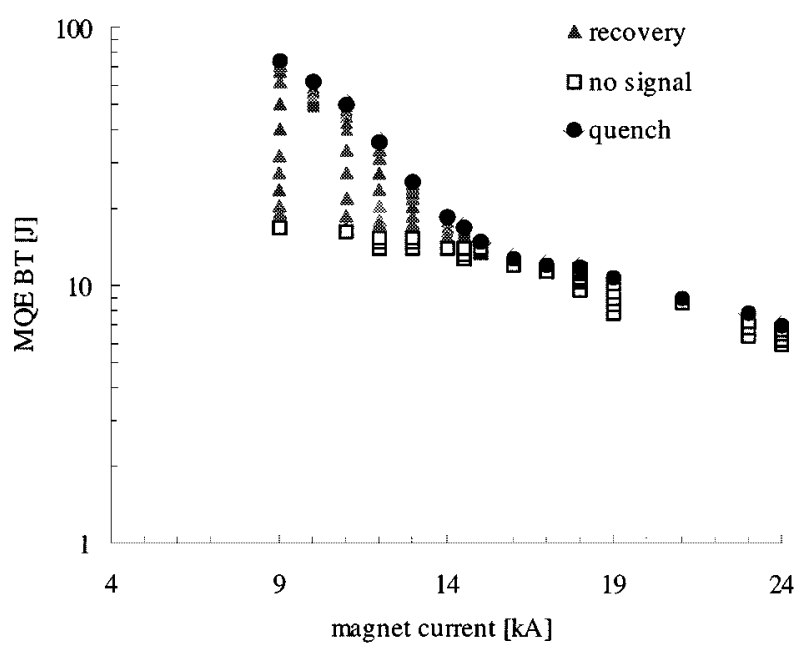

(a)

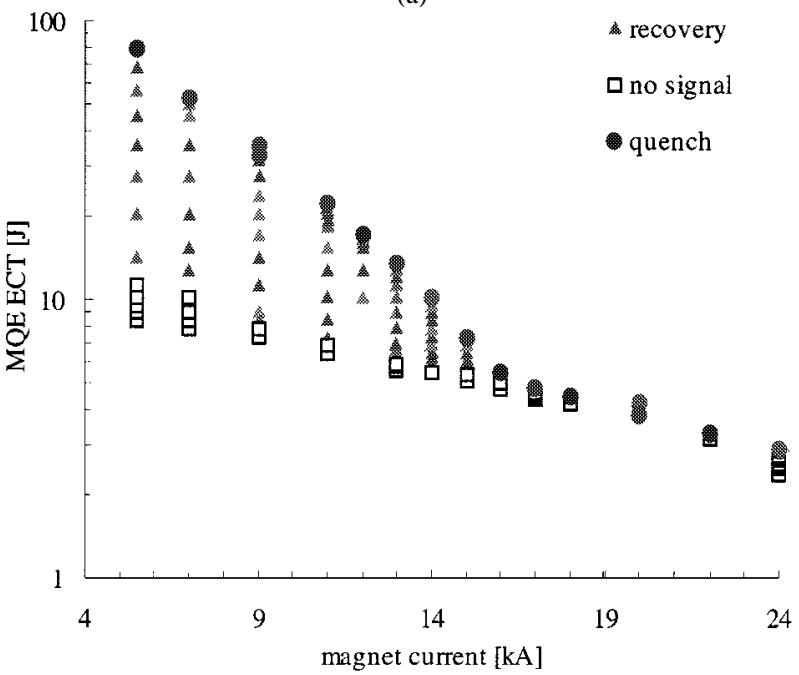

(b)

Fig. 6. (a) and (b) The minimum quench energies for the BT (above) and the ECT conductor (under) in B00 coil.

- Recovery: The coil voltage as well as the pickup coil signals, shows a current re-distribution.

- Quench: Fig. 6 shows that there is a well defined region where there is a recovery process. This can be understood considering that the cooling is more efficient than the heating i.e., the temperature of the superconductor doesn't exceed the sharing temperature. From the measured voltage tabs signals, a value for the characteristic recovery time, with a typically exponential behavior, of $4.2 \pm 0.1 \mathrm{~s}$. is determined for the BT conductor for operating currents up to $15 \mathrm{kA}$.

\section{CONCLusions}

The B00 model coil of Atlas is successfully tested up to $24 \mathrm{kA}$. It is suggested that a pick up coil wound around a Rutherford shape cable can be used as a sensor for magnet protection systems. It is found that the solenoid pickup coils situated above or under a layer of conductors detect the normal zone front in several layers. This measurement technique can determine the normal zone propagation in B00 model coil with reasonable accuracy in all the layers of a pancake, using only one sensor. A dominating longitudinal normal zone propagation velocity is found inside the BT and the ECT conductor between magnet currents of 5 to $24 \mathrm{kA}$. In the same current range the minimum quench energies of the $\mathrm{BT}$ and the ECT conductor are determined. It is found that there is a well defined region where the recovery process can be measured.

\section{ACKNOWLEDGMENT}

The authors express their warm thanks to all the members supporting the B00 tests. Special thanks goes to the cryogenic team of CERN.

\section{REFERENCES}

[1] P. Miele et al., "The ATLAS Magnet Test Facility at CERN," IEEE Trans. Appl. Superconduct., vol. 11, pp. 1713-1716, Mar. 2001.

[2] A. V. Dudarev, E. W. Boxman, H. H. J. ten Kate, O. P. Anaskin, V. E. Keilin, and V. V. Lysenko, "The B00 model coil in the Atlas Magnet Test Facility," IEEE Trans. Appl. Superconduct., vol. 11, pp. 1582-1585, Mar. 2001

[3] M. N. Wilson, Superconducting Magnets. Oxford: Clarendon Press, 1983, vol. 143.

[4] M. Scherer and P. Turowski, "Investigation of the propagation velocity of a normal-superconducting zone in technical superconductors," Cryogenics, pp. 515-520, Sept. 1978.

[5] N. A. Buznikov, A. A. Pukhov, A. L. Rakhmanov, and V. S. Vysotsky, "Current redistribution between strands and quench process in a superconducting cable," Cryogenics, vol. 36, pp. 275-281, 1996.

[6] F. P. Juster et al., "Stability and quench propagation velocities measurements on the 'Racetrack' mockup of ATLAS toroid coil," IEEE Trans. Appl. Superconduct., vol. 10, pp. 3434-34 343, Mar. 1999. 